\title{
ETAPAS METODOLÓGICAS DE UM PROCESSO CRÍTICO-REFLEXIVO SOBRE O TRABALHO DA ENFERMEIRA EM UNIDADE DE EMERGÊNCIA
}

\author{
Methodological Stages of a Critical-Reflexive Process \\ About the Work of Nurses in an Emergency Unit \\ Etapas Metodológicas de un Proceso Crítico-Reflexivo \\ Sobre el Trabajo de la Enfermera en Unidad de Emergencia
}

TâriaSolangeBosi deSouzaMagnago AnaLúraCardosoKrdhof CamemLúdaColoméBedk

\begin{abstract}
Resumo
Esteartigo relata o processo deconstrução de uma metodologia paradesenviver umprocesso cútico-reflevivoacerca

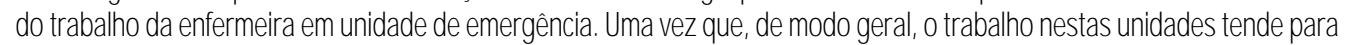

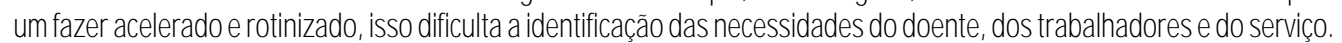
Oreferendal teórico-metodológcofoi a Teoria Sócio-humaristadeCapellae Leoperd. Os dados foramcoledados a partir da observação do campo e de seis encontros comenfermeras do senviço, mediados por uminstrumento quefoi drecionado paraacompreensãodbsujeitotrabalhador sobre o seu trabalho, aidentificaçãodas possibilidades edfiatdades nasua exeação ea reorganização db trabalho da enfermagem Com resultado, foi constrúdo umprocesso coletivoparaum'rnodbdefazer' caerfermagememunicadedeemergênáa.
\end{abstract}

Palamas-dhave Efermagem Enfermagemdeenergênda. Metodblogia Teorias deeffermagem

\section{Abstract}

This artide reports the process of construction of a methodblogy to develop a citica-reflexive process concerming the work of the nursein unit of emergency. Oncethat, ingeneral vay, the workintheseunitstends to $U$, afast andcaily make, that dffiault theidentification of the tien necessities of thesick person, of the workers and of the senice Thetheoretician-methodological referential was the Capella's andLeopard's Social-humarist Theory. The Sod data had been collectedfrom the dosenvion of thefiedd andfromsixmeting withnurses of thesenvice, medited foraninstrument that wes directedfor the understanding in
of thedligent of the possibilities and difficalties in their exeation and thereorganization of the work of then ursing. As result, was constructeda collective process for a 'vayto make' of thenursing in unit of energency.

\footnotetext{
Keymords=Nursing Energencynursing. Methodblogy. Nursing theories.
}

\section{Resumen}

Este artíalo relata el proceso de construccón de una metodología para desamollar un proceso cútico-reflexivo acercadel trabajodelaenfermeraen uridzddeerergenáa Unavezque demaneragenerd, el trabajoenestes uricades tiendeaunhacer rápidbydiario, eso dfíaltalaidentificacón delas necesidades del enfermo, delos trabajadores ydl senvicio. E referendial teórico-metodblógico fuélaTeoría Social-humanista de Capellay Leopardi. Los datos fuerón recogidos desde la obsenación de campo y de seis encuertros con enfermeras del senvicio, mediadas por un instrumento quefuedingidoperalacomprensiónde sujeto trabajador sobre su trabajo, la identificación de las posibilidades y las dificultades en su ejecurión y la rearganizacóndl trabajodelaefermería comoresultadb, fueconstridb un proceso colectivoparaun'modb dehacer' delaerfermería en unicaddeenergenia 
INTRODUÇÃO

Este artigo é umrecorte ampliado da dissertação intitulada "Una refleão cútica sobre o 'modo de fazer' da enfermeira perante o doente traumatizado grave emunidade de pronto-atendimento" ${ }^{1}$ e apre senta altemativas metodblógicas de trabalho diante das dificuldades apresentadas pelas enfermeiras que atuamemUhidades de Energênaia no sertido de buscar suporte às suas ações. Estas difialdades são evidenciadas no atendimento de pacientes emsituação de errergênaia, tendo emvista o somatónio de fatores como o déficit de trabalhadores de enfermagem no senviço, a insuficiência de reaursos técricos e materiais, a superlotação de doentes e tambémpela atua ção de enfermeriros envolvidos emumfazer acelerado e rotinizado, que prejudica a identificação e a definição das necessidades dos dbentes, dos trabalhadores e do senvço. É, principalmente, no que se relaciona aos trabalhadores de enfermagemque se obsenva a necessidade de buscar o significado de umconhedmento sistematizado para o alcance de umaiidado eficaz para o doente e, igualmente, satisfatónio para a equipe e para o senviço.

Face ao exposto e por perceber que as soluções para algumas dessas limitações estão dentro da esfera de governabilidade da enfermeira, foi proposta a realização de umtrabalho emconjunto comas en fermeiras de uma unidade de emergência, como objetivo de fazer umexerácio cútico-reflexivo sobre a realidade da assistência de enfermagem almejando que tais reflexões possibilitassema geração de novos conhecimentos. A expectativa é de que os conhecimentos produzidos sejamcapazes de senvir de instrumentos de intervenção no cotidiano da prática assistencial, pois se acredita que a enfermera, ao problematizar seu cotidiano, pode iniciar umproces so de descobertas que possibilitam uma 'desacomodacão', tanto na esfera pessoal, quanto na do serviço. Acreditando-se que o estudo contribuirá para o desvelamento do trabalho da enfermera, de limitourse como objetivo: apresentar a proposta metodblógica de um processo crítico-reflexivo realizado comenfermeriras de unidade de erergênaia.

REFERENCIAL TEÓRICO-METODOLÓGICO: A TEORIA SÓCIO-HUMANISTA ${ }^{a}$

Coerentes comessas considerações, buscourse perceber a tarefa de conhecer o 'modb de fazer' da enfermera, respaldando-se na Teoria Sócio-humanista de Beatriz Capella e Mria Tereza Leoperd a que de gemcono foco central - a valonização do sujeito (do- ente e trabalhador) e a valonização do trabalho ${ }^{2}$. Proa rourse enfocar o trabalhador enquanto objeto deste estudb, na perspectiva de umser humano inteiro, global, naquilo que ele temde sua sociabilidade e sugje tividade: ${ }^{139}$, buscando, por meio da análise do modo de fazer dessas enfermeras, as formas de sistematizar e dar visibilidade a esse trabalho.

Ao escolher a enfermera como djeto de estudo, foramfeitas adaptações às etapas metodblógicas pre conizadas pela Teoria Sócio-humanista, as quais estão voltadas ao sujeito portador de carênias de saúr de Ao se estudar essa teoria, percebeurse o seu potencial para refletir citicamente o trabalho emuridades de energênda, pois se entende que, para que o doente receba umatendimento de qualidade e seja visualizado emsua integralidade e, também para que os trabalhadores (enfermeiras) terhamo seu traba Iho valonizado, é impresaindivel 'revisitar' este trabaIho, dhandb-o pela rediação de una teoria que tam bémterha essa através da finalidade.

Neste sentido, os pressupostos e os conceitos da Teoria Sócio-humarista alicerçarama construção das etapas metodblógicas, as quais possibilitaramàs enfermeiras não só refletir sobre o que difialta e o que facilita o seu trabalho, mas tambémsobre o que é possível mudar no cotidiano.

Assim considerando a problemática desta unidade de energênda, alguns questionamentos nortearam o estudb, quis sejam Qul temsidb o 'modb de fazer' da enfermera na unidade de emergêndia perante o doente tramatizado grave? Oque pensamas enfermeiras sobre este 'nodo de fazer'? Quis são as possibilidades e os limites profissionais e institucionais para o desenvolvimento de umatendimento qualíica do e ético ao doente traumatizadb grave?

MARCO CONCEITUAL PARA A ELABORAÇÃO DA PROPOSTA

Onarco conceitul senve para diredioner ou giar o trabalho, atuandb como referênaia para o que deve ser dosenado, relacionado e planejado perante o tera de estudo, alémde ajudar a organizar as reflexões sobre o que está sendo vivendado. Nesse sentido, apresentam se, a seguir, os pressupostos e os conceitos propostos combase na Teoria Sócio-humarista e na experiência assistencial de una das autoras do estudo perante o doente trau matizadb grave na u ridade de energêna.

\section{Pressupostos básicos}

Pressupostos são as crenças e os valores do pesquisador que orientama elaboração de ummarco conceitual e auxiliamnas relações entre os conceitos propostos. Nessa perspediva, compreendese que 
- Odbente traumatizado grave é um“sujeito portador de carências de saúde", que necessita de atendimento imediato, sistematizado, eficiente e de qualidade, a fimce ter garantido o seu direito à vida, à ausência de iatrogenias e ao restabelecimento da sua autonomia;

- Aenfermera deve aliar a sua competênaia técnica à perspectiva humanística:139 durante o atendmento ao traumatizado grave, a fim de tomar menos dblorosa a situação vivida por esse sujeito e seus familiares;

- Ainstituição de um'rrodb de fazer' darte db dbente traumatizado grave, em unidade de entergência, possibilita ao trabalhador menor gasto de energia, bemcono menor nível de estresse e ansiedade; - Aerfermeira ao refletir sobre oseu catidano enedar seu trabalho por uma teoria, passa a construir corhedimertos, pernitindb não só se tranरformær, næs tanbémtransformar a sua realidade de trabalho; - O processo de construção cooperativa do 'modo de fazer' da enfermera possibilita tanto a construção de ummodo de fazer melhor quanto uma niior adesão do gupo a esse nodo de fazer.

\section{Conceitos}

Para o desennolvimeto do processo reflevivo, serviramcono referênia os conceitos de ser humano, tra balho, instituição de saúde, processo de trabalho em saúde, processo de trabalho emenfermagem enferma gem sujeito trabalhador de enfermageme sujeto portador de crências de saúde apresentados pela Teoria Sócio-humanista Alémdesses conceitos, proarourse delinear algumas considerações soldre o 'rodo de fazer' da enfermeira na unidade de energência, norteadas pelas experiênaias e valores de una das autoras com vistas a una (re)orientação desse 'nodb de fazer'.

Na Teoria Sócio-humanista, o ser humano é entendido com:

umser natural, que surge emuma natureza dada, submetendo-se às suas leis para sobre viver. É parte dessa natureza, nas não se confunde comela, pois a usa, transformando-a conscienterente, segundo suas necessidades. Nesse processo, se faz humano e passa a construir a sua história, se fazendo histónico $0^{2: 142}$.

Este ser humano está emconstantes relações com autos seres humanos, que fazem parte do anbiente ao seu redor. É umser de relaçōes que influencia e sofre influências emsuas atitudes e comportamentos, compossibilidades de crescer e completar-se com autros seres humanos. Está inseridb emummeio com o qual interage, realizando constantes trocas, transformando-o e, já que é parte integrante dele, sendo tambémtransformado.

O"sujeito portador de carênias de saúde", o dbente trau matizado grave, é umser humano que, em seu perarso de vida, por alguma circunstânda, se encontra diante de uma condição de agravo à saúde, ocasionada por feimento grave, necessitando de dendimento imediato, sistematizado, eficiente e de qualidade Utiliza os seniços de emergênia, submetese à intenvenção dos profissionais de saúde coma finalidade de restabdecer as suas funções vitais e a sua autonomia e, ainda, ficar isento de iatrogenias.

Neste estudo, traumatismo grave será entendido como toda a lesão que assume caráter de extrema gravidade, não só pelo comprometimento inediato das funções vitais, mas tambémpela freqüente associação a traumatismos múltiplos que podemlevar ao óbito, caso não seja imediatamente proporcionada uma assistêndia adequada.

Na Teoria Sócio-humanista o trabalho é conside rado como a:

ação do ser humano no desenviluimerto de seu percurso histónico, aliando sua materialidade (força física) à sua capacidade de pensar e rea gir, emsuas relações comoutros homens, para atender a sua necessidade natural de solbrevivênaia, determinando una outra forma de fenômeno - o trabalho, que consiste nummodb dferenciado de intervenção sobre a natureza, pela definição de projetos, sua implementação, realização de produtos, para alémde si mestro e da natureza, isto é, pela recriação da natureza:143.

Otrabalho da enfermeira, neste estudb, foi caracterizadb como umconjunto de açōes que compreande não somente os procedimentos técricos e os equipa mentos empregados pela enfermera na assistêndia ao doente traunatizado grave, mas tanbéma aplica ção do saber profissional na resolução dos problemas identificadbs, de forma a possibilitar a geração de ações transformadoras no trabalho emsi, ou mesmo nas veriadas airanstânaias que o cercame o determinam No trabalho, o 'modb de fazer' da enfermera se dínge para váios objetos como, por exemplo: a educação, a gerência, a assistêndia emsi, entre outros. Ee pode ser desenvolvido de forma sistenática ou assistenática. Neste estudb, o 'modb de fazer' da enfermera se constituirá no seu método de trabalho, tendo como ele mentos norteadores a finalidade, o objeto, os instru mentos e a força de trabalho empregados para o seu desenvolvimento. 
A forma como o seniço está organizado imprime uma qualidade na assistência, portanto, no 'rrodo de fazer', sendo que há diferentes formas de compreender a organização do trabalho. As autoras da Teoria Sócio-humanista entendema organização do traba Iho da enfermagemcomo o modb comb os trabalha dores de enfermagem dispõemo seu trabalho e fornecema base para o trabalho de outros profissionais na instituição de saúde, emrelação aos tempos, movimentos e objetos necessários à assistêndia da saúdel:153. Eas separam conceitualmente, a organização das relações de trabalho na enfermagem en tendendo que as relações de trabalho:

são relações que se dão no exerácio da profissão: internamente, coma equipe de enfermat geme, extemamente, comoutros profissionais, o sujeito portador de carências de saúde e a instituição. As relações de trabalho referemse às relações pessoa/pessoa e pessoa/djieto: ${ }^{2} 154$.

Já, outros autores entendemque, na organização do trabalho, alémcla divisão do trabalho (d̛visão de tarefas entre os operadores, repartição, cadêndia e, enfim o modo operatónio prescrito), há a divisão de homens (repartição de responsabilidades, hierarquia, comandb, controle, entre atros) 3:125, que aparece como umcomplemento necessário, pois enquanto:

a divisão das tarefas e o modo operatónio indtamo sentido e o interesse do trabalho para o sujeito, a divisão de homens solidita, sobretudb, as relações entre pessoas e mobiliza os investimentos afeivos, o anor e o ódio, a anizade, a solidariedade, a confiança, entre outros 3:225.

Esses 'investimentos afeivos' são importantes ao se considerar a possibilidade de construir emconjunto una proposta de trabalho, emumprocesso de ca minhar juntas. Processo esse emque as interações, as análises e as reflexões realizadas pelo grupo subsidiariama construção coletiva do 'rrodo de fazer' da enfermeira, na busca por uma assistência sistematizada, eficiente e de qualidade, ao doente tra matizadb grave, por meio de açães de manutenção, de reparação e de encaninhamento.

A Teoria Sócio-humanista preconiza a utilização dessas ações no momento do planejamento da assistência de enfermagemjunto ao sujeito portador de carências de saúde. Para as teóricas, as ações de manutenção são aquelas que cấ sustentação à vidą:166, levendb-se emconta os hábitos, os costumes e as cren ças do sujeito portador de carênaias de saúde As ações de reparação são açães de intenvenção àquelas que se constituemobstáculos à vida; as ações de orienta. ção são as que visamao retomo gradativo da a utonomia deste sujeto e a orientação ddle e de seu familiar sobre os procedimentos que poderão ser necessários: ${ }^{2}: 166$. E as ações de encamimamento referemse às solicitações que o enfermeiro deverá fazer junto a outros profissionais, por ser este umtrabalho interdependente, coletivo e de autonomia relativa'.

Para este estudb foi dada êtase res ações de nanut tenção, de reparação e de encaminhanerto para a organização do trabalho da erfermeira, sendb levados em conta os elementos do processo de trabalho emerfermagem, de rrodb a atender à firalidade a que se propõe

Neste corteto, considerandb o dgjeto, a finalicade, os instru mertos e a força de traballo no cotidano da erfermera de unidade de urgênda e entrgênda, entendese que

- ações de manutenção: consistemnes ações quea enfertera efetua no seu cotidano de trabalho, que, conforme a aveliação do gupo, podemser nartidas; - ações de reparação: consistemna redaboração da ação que foi negodiada como grupo, para a sua implenertação no cotidano do trabalho da enfermeria - ações de encaminhamento: referemse à elaboração de umdoaumento de encaminhamento de situações que predisamser reparadas, mas estão fora da governabilidade da enfermeira, e que, portanto, são encaminhadas à direção de enfermagempara conhedimento e providências.

Para un n thor compreensão da relação dos concẹtos utilizabs, fai constrúcba a figura representada ra página segir.

Representação gráfica do marco conceitual

Nb desenvolvimerto de umprocesso de canimher jun tas para o 'nodb de fazer' da enfermera (figura 1) ante o dbente traumatizado grave, emunidade de energênda perpassamas caraderísticas histónicas do níve sodal, do forma e do particular. Apesar da importânda de todos os níveis, neste processo reflevio foi dada naior êfase ao ríd partialar eao formম, pais são ndes que as relações do trabalho da enfermerira se condetizam

Sendo assim, o processo de caminhar juntas partiu inicialmente, de una caminhada solitária a seja, da doservação de campo das vivêndias no diazada de trabaIho da enfermera (MMDO), ra unicade de energênàa A partir dessa dosenação, foi iniciada una caninhada coletiva comos sujeitos do estudb, que levou a reflexões sobre o 'rrodb de fazer' ca enfermera, a partir da prática cotidana institúća na unidade

As reflexões, enbasadas nos conceitos de ser hut mano, enfermeira de unidade de emergência, trabaIho, trabalho da enfermeira e organização do trabaIho, conduriramo grupo a adotar ações de manutenção, de reparação e de encaminhamento. Tais ações 
Figura 1:

Desenvolvimento do processo de caminhar juntas para um 'modo de fazer' da enfermeira perante o doente traumatizado grave em unidade de emergência.

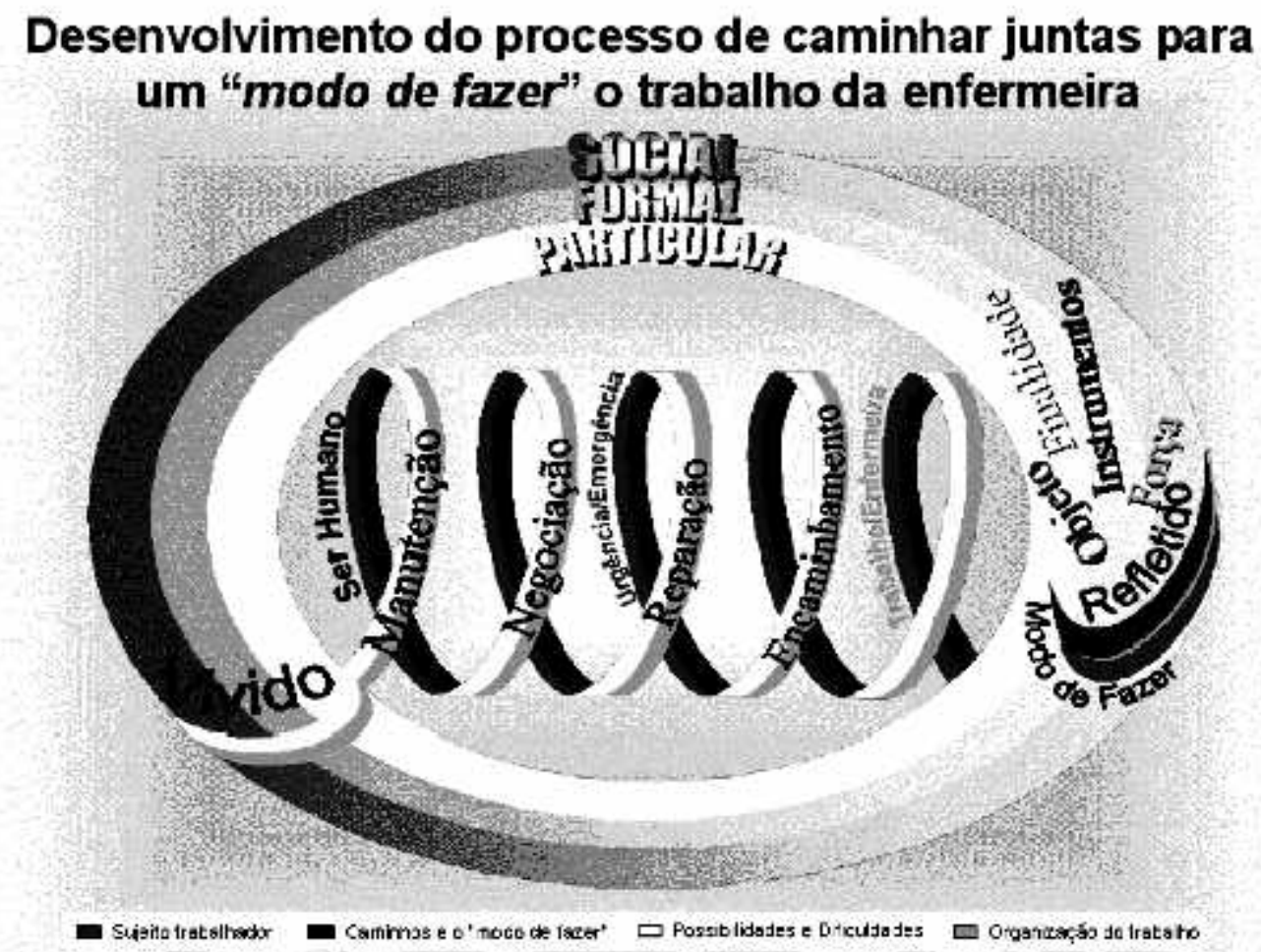

Representação gráfica do Marco Conceitual

forammediadas por outra ação, a de negociação, objetivendo dhegar a umconsenso sobre o 'modo de fazer' da enfermera (R⿴囗十⺝IDO), ou seja, a qual dojeto de trabalho se destina? Qul a finalidade? Quis os instrumentos a seremutilizados? Qua a força de trabalho necessánia para a prestação de una assistêndia humanizada e de qualidade?

O processo de caminhar juntas foi representado pela forma helicoidal por ser considerado contínuo, conjunto, aberto e dinâmico. Este processo contem plou várias fases que se complementarame se interligaramconstantemente. As fases foramtradzidas, na prática, por momentos intitulados de "compreensão do sujeito trabalhador", "descoberta de caminhos e observação do 'modo de fazer' da enfermeira", "identificação das possibilidades e das dificuldades na exeação do trabalho da enfermera" e "o caminhar para una reorganização do trabalho da enfermeira".

$\mathrm{Nb}$ deserrolar dessas fases, foram djjeivedos respedivamente: ter una melhor compreensão da enfermera que atua emunidade de energência, identificar qual é o trabalho da enfermera nessa unidade; refletir sobre o que dfiautta e o que fadilita o seu trabalh, quais as possibilidades de reestruturálo e, por útimo, com base nessas reflex̃es, negodiar, como grupo, quais das ações desenvolvidas pelas enfermeras poderiamser manticas, quais necessitariam de reparação e, entre essas, quais poderiamser reparadas por elas próprias e quais predisariamser encaminhadas.

\section{METODOLOGIA PROPOSTA PARA O \\ PROCESSO CRÍTICO-REFLEXIVO}

Ao elaborar a Teoria Sócio-humanista, as teóricas desenvolveram una metodologia, que elas entendem comr um'rrodo de fazer' o trabalho de enfermageme a explidtaramemseis etapas preliminares ${ }^{2: 163}$, para serem desenvolvidas junto ao sujeito portador de carênias de saúde, quais sejam

(1) Identificação do portador da necessidade;

(2) processo de viver, ser saudável e adbecer,

(3) projeto cooperativo de trabalho;

(4) negociação e implementação;

(5) processo de avaliação e/ou replanejamento;

(6) possibilidades e limites institurionais e legais

Comb o dgjeto deste estudb é o sujeto trabalhador, adequamos a metodologia de Capella e Leopard, de forma a contemplar as necessidades das enfermeiras 
da unidade de emergência. As etapas foramentão subolvididas de acordo como esquerta demonstrado, a seguir, no processo de enfermagem
Para colocar emprática esta proposta, o projeto passou inicialme pela Comissão de Ensino, Pesquisa e Extensão ( $\oplus P E)$ e pelo Comitê de Ética do Centro

Figura 2:

Diagrama das etapas metodológicas do processo crítico-reflexivo.

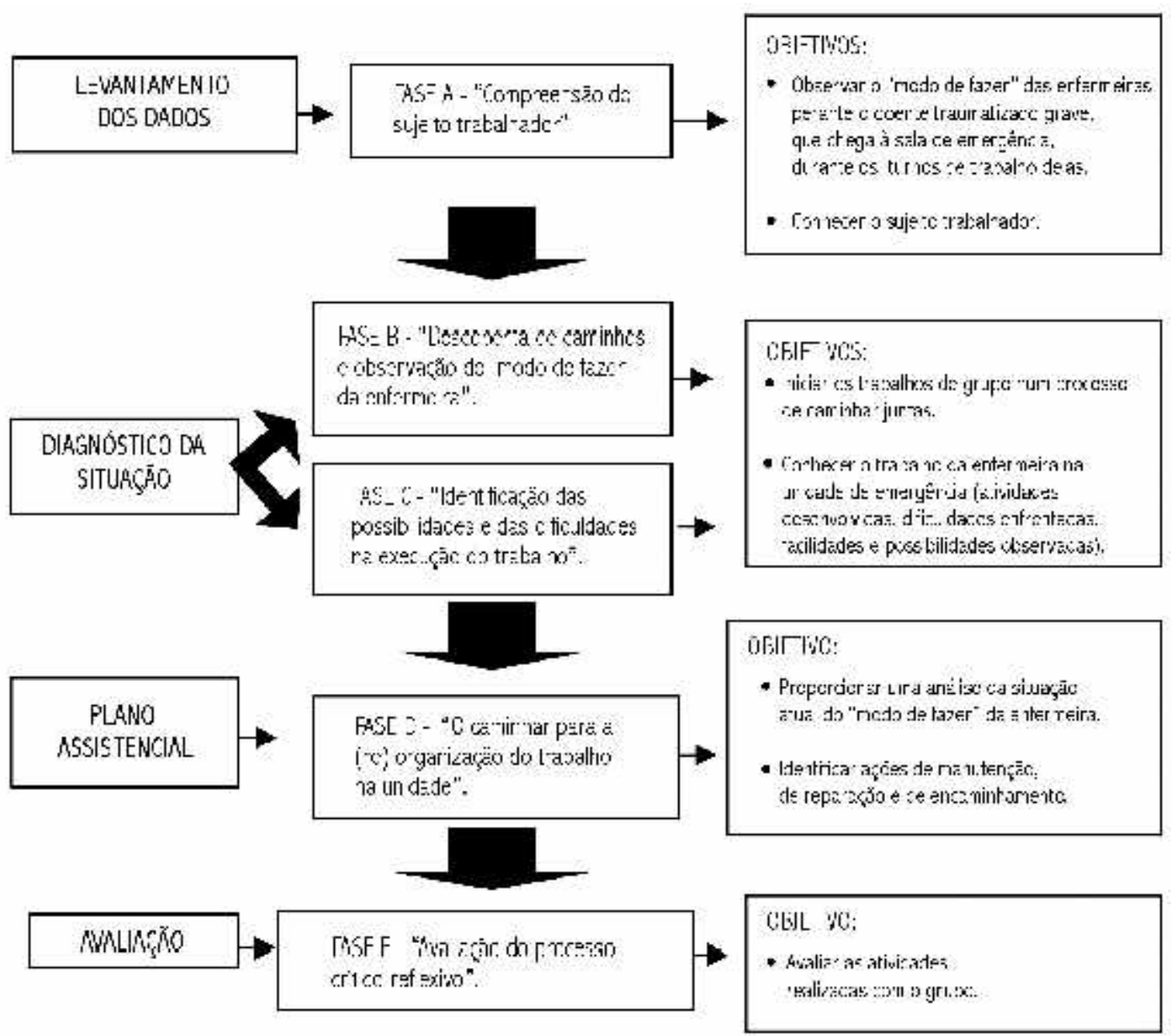

de Gências da Saúde da Instituição pesquisada, recebendo parecer favorável. Após, reelizamos contato comas enfermeras da unidade de entergêndia, e foi assinado o Termo de Consentimento Live e Esdaredido, conforme preconizado pela Resolução 196/96, do Conselho Nacional de Saúde, no qual os sujeitos concordaramemparticipar do estudb.

Para a coleta de dados, construímos um instrumento (Anexo 1), subdividido emcinco fases e aplicado, parte dele, na obsenvação de campo, como questionánio e, outra parte, nos seis encontros realizados como grupo, como entrevista coletiva. Participaram destas atividades quatro (100\%) enfermeiras da unidade de emergência.

\section{O DESENVOLVIMENTO DAS ETAPAS METODOLÓGICAS}

Neste item consta especificamente a descrição do desenolvimerto de cada una das fases, como dijetivo de ajudar o profissional que por ventura, queira utilizar estas etapas emoutro estudo. Portanto, não serão disautidos os dadbs encontrados emcada una delas.

\section{FASE A}

Compreensão do sujeito trabalhador

A operacionalização dessa fase se deu no período de observação de campo por meio da própria observação do trabalho na unidade de energência e do preendhimento de umquestionário (Anexo 1 - A1 
e A2) pelas enfermeras. Tal questionánio possibilitou traçar o perfil destes sujettos trabalhadores e obter dados comrelação ao trabalho/saúde/doença dos mesmos. Também durante o primeiro encontro com o gupo, foramobtidos dados que contribuíramnas reflexões sobre os adhadbs no questionánio e perceber que o ritro de trabalho pode provocar conseqüências negativas nas enfermeiras devidas ao desgaste viveniado por elas no seu cotidiano laboral 5 .

\section{FASE B}

Descoberta de caminhos e observação do 'modo de fazer' da enfermeira

Para operacionalizar esta fase, durante o segundo encontro, foram utilizadas como subsídio para as discussões do grupo as questões da Fase B do instrumento emanexo. Emumcartaz, foramlistadas as atividades desenvolvidas pelas enfermeiras na unidade de energêndia, e enfocadas questões de cont era percebido esse trabalho e qual a metodblogia utilizada para desenvolvê-lo. Combase na Lei do Exerácio Profissional, foi identificado e refletido sobre o que era e o que não era trabalho da enfermera. Ao final, construitse umnovo cartaz listando as atividades espeáficas da enfermeira na unidade de emergênaia.

\section{FASE C}

Identificação das possibilidades e das dificuldades na execução do trabalho

Esta fase fai desenvivica durante o terceiro, quato equinto encontros como gupo. Nb terceiro encontro, para introduzir as reflexões da Fase Cdo instrumento de coleta de dados (Anexo 1), foramrealizadas leitura e disaussão do texto O micropoder no processo de trabalho dentro da estrutura hospitalar: vivendiando uma históniæ. Após, foi resgatado o cartaz solbre a lista de atividades indicadas para una enfermera de unidade de emergêndia, centrando as reflexões nas difiauldades encontradas para o desenvolvimento do trabalho. Para esta atividade, foi entregue umroteiro gia (Anexo 2), para ser respondido individualmente e depois, disatido coletivamete

Nb quarto encontro, foi enfocada a questão $1 \mathrm{da}$ Fase Cdo instrumento (Anexo 1), como propósito de fazer umlevantamento das facilidades que o espaço hospitalar oferece para o desenvolvinento do trabalho da enfermeira (Anexo 3), tendo por base o cartaz de atividades construído no segundo encontro.

$\mathrm{Nb}$ quinto encontro, combase nos achados durante a doserveção de campo, foi dranatizadb o đtendimento a umpaciente que dhega à uniclade de energênaia. $A$ partir dessa atividade, as enfermeiras receberamum roteiro (Anexo 4) para que listassemos pontos frágeis, os pontos fortes e as implicacões éticas percebiclas no atendimento dramatizado. Ao final, os achados foram disatidos coletivamente, e foramconstruídos os conceitos de enfermera de unidade de energência, trabalho e doente traumatizado grave, enquanto produção/concepção deste gupo de enfermeiros tendo emvista o seu contexto de atuação.

\section{FASE D}

O caminhar para a (re)organização do trabalho da enfermeira

Esta fase fai qperaciondizada no seto encontro e teve por dgjetivo possibilitar ao gupo, por intemédio da análise ontica sobre o apreendidb até aquele momento, adbtar açães de mantenção, de reparação e de encanimhanerto (Anexo 1), comvistas às possibilidades obsenvadas. A disaussão foi encaminhada a partir das reflexões anteriores, que envolverama tomida de consciência das enfermeras sobre seus trabalhos, incentivandb-as a visualizarem(questão 3 da Fase Q quais eramas possibilidades de enfrentamento das dificuldades encontradas no cotidiano.

Aestratégia para alcançar o proposto era, combase emtodas as disaussões levantadas durante os encontros e nos elenertos do processo de trabalho emenfermagem (djjeto, finalicade, instrumetos e força de trabalho), fazer as enfermeras negociarem no grupo: quais acõoes executadas por elas, na unidade de etrergêndia, que deviamser marticas; quais ações deviamser reparadas, considerando que essa reparação estava dentro da governabilidade delas; quais ações necessitavamser reparadas, porémestavamfora da sua governabilidade e, portanto, seriamencaminhadas corro sugestão aos setores competentes.

\section{FASE E}

Avaliação do processo crítico reflexivo

Ao find do seto encortro fai entregue umquestionánio (Aneo 1) como propósito de coletar informaçóes acerca do que significou para cada enfermera participar desse processo; se a metodblogia proposta senviu para tomar consciênia de seu trabalho, dos prodlenæs vividos e da possibilidade de transformação da realidade a partir da

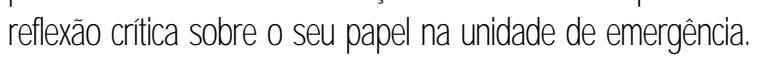

\section{CONSIDERAÇÕES TEÓRICAS}

SOBRE O PROCESSO

Ao longo da caminhada, embusca da construção do processo de caminhar juntas, foi utilizada a problematização do cotidiano como desencadeadora das disaussões. As múltiplas reflexões realizadas com o grupo de enfermeiras permitiramemergir mitos 
dados que potencializampara algumas transformações no cotidano de trabalho na unidade de entergênda. Essas reflexões tiveramsuporte nos conceitos da Teoria Sóciohumarista, prinaplinerte no que dz respeito ao processo de trabalho emenfermageme seus elementos, ou seja, o djieto, a finalicade, os instru mentos e a força det trabalho.

Ao estudar a Teoria Sócio-humarista, ficou evidente que o enfoque preconizado pelas teóricas era perfétamente aplicável à unidade de energêndia Entbora a própria teoria destaque que os dojetos de trabalho da enfermera são os conpos e a consciênia dos indvíduos ${ }^{2}$ e, também a organização do trabalho², houve uma limitação para desenvolver a proposta ora pretendica. Qu seja, as etapas metodblógicas propostas pela teoria estão direcionadas ao sujeito portador de carências de saúde No entanto, para o desenvivimento deste estudo, havia necessidade de uma seqüência metodológica voltada ao sujeito trabalhador direcionando as reflexões sobre a organização do trabalho da enfermera.

Surgu daí, una officuldade, mas tambémumgrande desafio: adequar as etapas metodblógicas de forma a contemplar a valorização do sujeito trabalhador e a orgarização do trabalho. Para isso, fai necessánio ver as colegas enfermeiras cono sujeitos trabalhadores, e não apenas como profissionais, como a rotina nos impõe; identificar qual era o trabalho delas na unidade de emergência; quais eramas dificuldades para realizar tal trabalho; eainda, idertificar que possibilidades das sugeriam pera unæ (re)criação da prática assistendal.

Acreditase que, entbora se terha adaptado a teoria a umobjeto de estudo diferenciado, a trabalhadora enfermeira, não houe prejúzos, nas sima possibilidade da ampliação da sua aplicação no campo práticoassistencial. Conduiurse que a adaptação feita ao trabalhador pode ampliar o foco da teoria e fazer tanbém desse personagem umimportante objeto de estudb a ser contempladb.

No decorrer do processo reflevivo, as etapas metodológicas construídas contribuíram para o entendimento de que a Enfermagem é uma prática sodial cooperativa e coletivaz:153. Contribuíram também para que as enfermeiras, com trabalhadbras que atuamem unicade de energência adbtassemaçõ̃es de manutenção, de continuidade da vica, e açōes de reparação a tudb que se constitui obstáalo à vida do doente traumatizado grave $\mathrm{Da}$ mesma forma, senviram para a tomada de consciêndia e para o entendimento do doente, em umsegundo momento, com sujeito das ações de saúde a ele ministradas emuma perspectiva de reconstrução de sua autonomia.

A pertir dá, a compreensão do processo trabalho na uridade condriu o gr po a pensar o seu fazer ever um 'nodb de fazer' mais humarizado, tanto para o sujeito trabdhador quento para o syjeto hospitdizad, pais have naior coesão nas ações a seremencaminhadas e no trabalho que se seguiu a esse estudb.

Por fim lançando um olhar sobre os pressupostos e sobre os conceitos traçados para entender o que representou o processo de caminhar juntas, surgiu a idéia de que tanto o trabalho da enfermera, quanto o sujeito-objeto da nossa prática não podemser vistos isoladamente, pois eles são interligados e interdependentes.

\section{Referências}

1. Magnago TSBS Una reflexão crítica sobreo 'rodo defazer' da efermera peranteodbertetra matizadbgraveem nicadedeprontoatendimento. Dissetação[Mestradbem Erfermagem]. Forianópolis: UFS; 2002. 184p

2. CapellaBB, Leopard, MT. Teoriasódio-humarista In Leopard, MT. Tearias de effermagem instrumertos paraa prática Florianópolis: Papa Livos, 1999, p. 137-71.

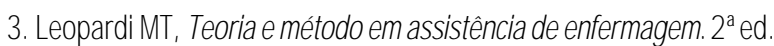
rev. eampli. Forianópolis: Ed Soldasoft; 2006.
4. DejoursC, Aboduchali E ltinerárioteónicoempsicopatdogiadb trabalho. In: Dejaurs C, Jayet C Psicodinâmica do trabalho: contribuições da Escola Dejarianaàanálisedarelação prazer, sofinertoetrabalho. São Paulo: Atlas, 1994. 145p.

5. Souza MDO, Lisboa ML Ritme de trabalho: fator de desgastepsíquico da enfermera. EscAma Nery RevEnferm 2005 ago; 9(2): 229-36.

6. NetscheE Omicropoder noprocesso detrabalho dentroda estrutura hospitalar: vivenciando una história. RevBras Erferm 1996, ju/set; 49(3): 373-90.

7. FreireP.Pedagogiacaa tonomia saberes necessáriosà prática edurativa 11ㄹed SãoPalo: PazeTera, 1996. 165p. 


\section{Nota}

a ATeariaSócio-humaristanasceudeumetensotrabalhodeprática zssistendad realizadoem mhospital universitániode Santa Catarina. Deacordo comleopardi(13108), estaéumadas propostasteóricas queilustramdiversas correntes depensanento nae fermageme apresenta umconjunto deproposições para a organização geral dotrabalho.

\section{Sobre as Autoras}

\section{TấaSolangeBosideSoura Magrago}

Erfermera. MestreenEnfermagempela U hiversidadeFederal de SartaCatarina(UFSASO. Docentedo Departanertode Erfermagem da UniversidadeFederd deSantaMeria (UFSMRS). Datorandado NúdeodePesquisaE fermagemeSádedbTrdbalhador- NPENST, daEscoladeErfermagemAmaNery- UfPJ.

E-mail:tragnago@teracombr.

\section{Aralúcacardosokindhof}

Enfermera DatoraemFilosofiadaEfermagempdaUFSC Docertedb DepartametodeErfemagemdaUFSC E-nail:kirdhof@teracombr.

\section{GrmemlúdacolonéBedk}

Evermera DoutoraemFilosofiadaE EfemagemdaLFSC Docertedo LepartamertodeErfermagemdaUFSM E-mail:camenbedk@smail.uśrmbr. 
ANEXO 1

Fases de desenvolvimento do 'processo de caminhar juntas'.

FASE A

A. 1 - Identificação

Codinome ldade

Háquartos anos exerceaprofissão deerfermeira? Cargooufunção:

Háquantosanostrabalhanainstitucăo?

Quisos setores emquejátrabalhou?

Trabalha, notud setor, háquentotempo?

Mbtivo deestar trabalhandb nessesetor. ( ) transferênciaa pedidb ( ) transferênia por indicação da direção ( ) atro

Comoocorreusuaintegraçãonosetor: foi apresentadoà equipe?

Recebencapacitaçãoespećficaartes deiniciar as atividades?

Auricadeeainstithiçãoforammostradasavocê?

Foi informadadamissão, filosofiae políticas dainstithição?

Vocêjátrabalhouatteriometeemuridadedeurgêncialenergênda?

Vocêparticipoudealgumarso espećficoemurgêndia/energênda, nosútinnos cincoanos? Qal (is)?

A. 2 - A vida profissional e a saúde/ doença do suj eito trabal hador

Seutrabalholhecausa ( ) estresse ( ) ansiedade/ angústia( ) prazer ( ) degria ( ) raiva( ) atros sentimentos dessegênero? Qais? Vocêpossi algumproblenædesánde? Qdal (is)?

Nbsútimos cincoanos, precisouficar afastadadotrabalho? ( ) Sim( ) Náo

Emcaso aimativo, tevealgumarelaçãocomotrabaho? ( ) Sim( ) Nâo

Emcaso afimativo, emquemedidasuadbença afeta seutrabalho?

Enquemedida suadbença ofetasuavidafariliar esuavidaretiva:

Vocêtemautroemprego? Howedgumftoimportante, relacionadbasuasaúde, quevocêgostariader latar?

FASE B. Descoberta de caminhos e observação do "modo de fazer" da enfermeira

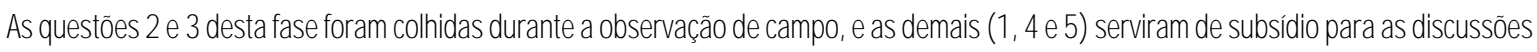
nosegundbencontro.

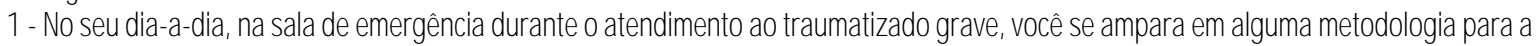
assistêniadeerfemagen? Qal?

2- Qa éotrabalho da enfermeira na uridadedeurgêndia/energênda? Expresse o por meio deanotações das atividades realizadas dariamente, duranteumperíodb deaincodis:

3- Quis as ações quevocêredizou nos útimos ainco dizs, perarteo dbentetraumatizadb gravequechegouà saladeetergência?

4-Conv vocêinterpretaotrabalhodaeffermeranauridadedeemergênda?

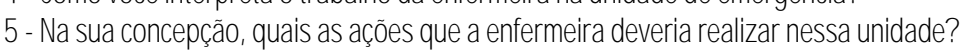

FASE C. Identificação das possibilidades e das dificuldades na execução do trabalho

Osquestionametos destafeseseniramdesubsídos paraas disassões doterceiro, quarto equintoencontros.

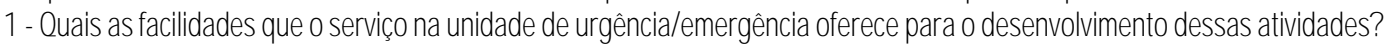

2-Quis as difialdades quevocêencontraparadesenviver as dividades listadas?

3- Enseuentender, conva enfermagempoderomper comas dfiauldades inerentes aoseutrabalho? Quis as possibilidades quevocê apontaparaa viabilização dessetrabalho?

FASE D. 0 caminhar para a (re)organização do trabalho da enfermeira

Estafesefoi desendviclanosetoencontro, emqueforamredizadas:

1. Ações demantenção; 2. Ações dereparação; 3. Aşões deencanimhanetto:

FASE E. Avaliação do processo crítico-reflexivo

Asquestões destafaseforametregues às enfermeras nosetoencontro.

Oquesignificouparavocêaexperiênciavivichemgrup?

Oaue, nessaexperiência, nais lhedhamouaztenção?

Aexperiênia vividacomo gnypo represerta uma possibilicadedeaprimoramertoe desenvolvimento desues competências pessoaise profissionais? ( ) Sim( ) Nấ Porquê?

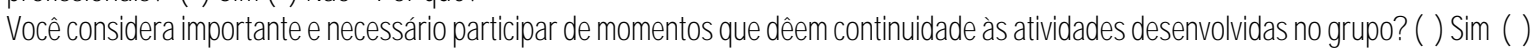

Náo Sepositivo, sugramodalidades:

Vocêconsideraqueam todblogiapropostanesseestudosenviupara:

1- Tomar consciênciadeseutrabalho, desuaformadepensar eatuar conoprofissional? ( ) Sim( ) Náo Por quê?

2- Tonar consciênadados problemas vividos pelogn po? ( ) Sim( ) Náo Por quê?

3- Aançar numbaumatendimentomais humanizadbedequalidade? ( ) Sim( ) Nấ Porquê?

4- Entender omododefazer otrabalhodae fermeracomo uminstr mentodetrabalho quedeveser colocadbempráticapela equipe?

( ) Sim( ) Náo Porquê? 


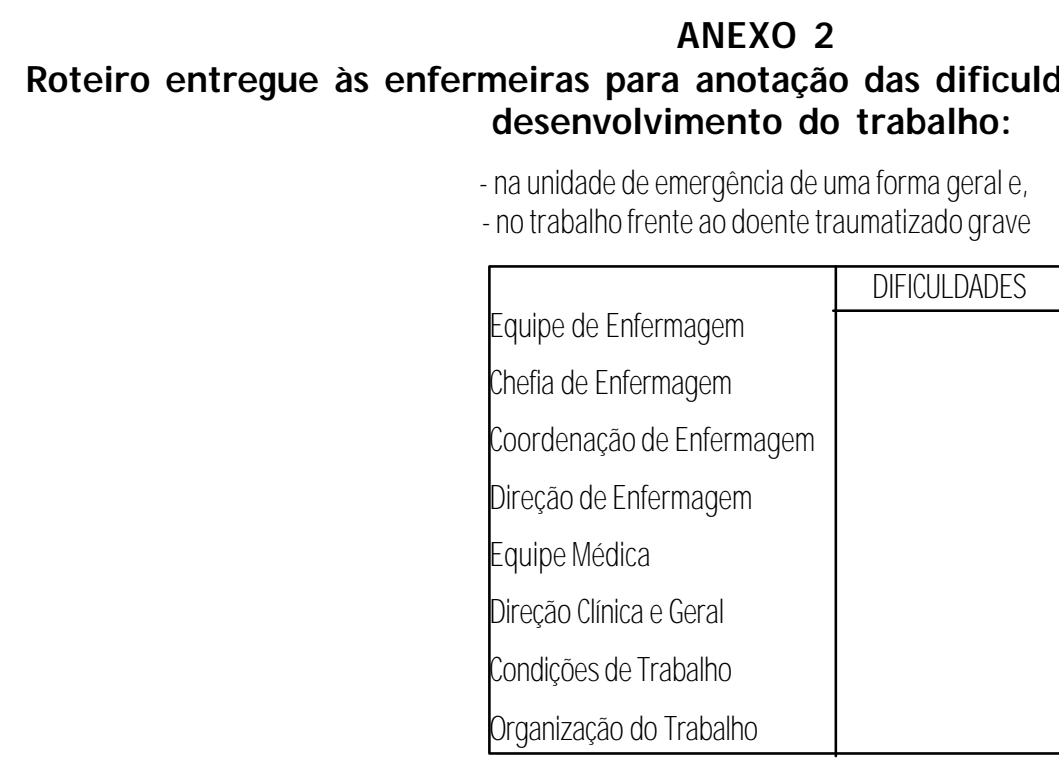

\section{ANEXO 3}

Roteiro entregue às enfermeiras para anotação das facilidades encontradas para o desenvolvimento do trabalho:

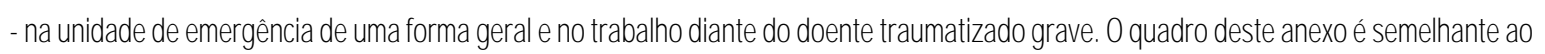
do anexo2, sendbaúricaalteraçãoatrocadapalava'difialdades' pelapalava'fáilidades'.

\section{ANEXO 4}

Roteiro entregue para às enfermeiras para anotação dos pontos fortes, pontos fracos e implicações éticas observadas no atendimento durante a dramatização:

\begin{tabular}{|l|c|c|}
\hline PONTOSFORIES & PONTOSFRACOS & IMPUCAÇ̃̃ESÉ|lCAS \\
\hline & & \\
& & \\
& & \\
\hline
\end{tabular}

